\title{
R EVUE
}

\section{INTERNATIONA LE}

DE LA

CROIX-ROUGE

ET

\section{BULLETIN INTERNATIONAL}

DES

\section{SOCIÉTÉS DE LA CROIX-ROUGE}

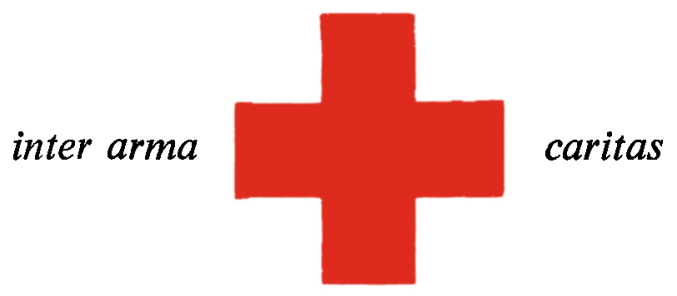




\section{COMITÉ INTERNATIONAL DE LA CROIX-ROUGE}

\section{à $G$ enève}

MM. MAX HUBER, docteur en droit, ancien président de la Cour permanente de justice internationale, president d'honneur (1923) 1

PAUL RUEGGER, docteur en droit, ancien ministre de Suisse en Italie et en Grande-Bretagne président ( 1948 )

JACQUES CHENEVIERE, docteur ès lettres h. c. (19I9)

M110 LUCIE ODIER, ex-chef du Service des infirmières-visiteuses de la Section genevoise de la CroixRouge suisse (1930)

MM. CARL J. BURCKHARDT, docteur en philosophie, ancien ministre de Suisse en France (I933)

ÉDOUARD CHAPUISAT, docteur ès lettres h. c. (1938)

ALEC CRAMER, docteur en médecine, colonel ( 1938 )

MARTIN BODMER, docteur en philosophie h. c. (Ig40)

ERNEST GLOOR, đocteur en médecine, vice-président (I945)

RENÊ VAN BElRCHEM, docteur en droit, banquier (I946)

LÉOPOLD BOISSIER, docteur en droit, professeur à l'Université de Genève, ancien secrétaire général de l'Union interparlementaire (1946)

PAUL CARRY, docteur en droit, professeur à l'Université de Genève (r946)

EDMOND GRASSET, dacteur en médecine, professeur à l'Université đe Genève, directeur de l'Institut d'bygiène (1946)

HENRI GUISAN, général, ancien cornmandant en chef de l'armée suisse (r948)

ALFREDO VANNOTTI, docteur en médecine, professeur à l'Université de Lausanne (I949)

RODOLFO OLGIATI, ancien directeur du Don suisse (1949)

M"le MARGUERITE VAN BERCHEM (I95I)

MM. FREDÉRIC SIORDET, avocat, conseiller du Comité international de la Croix-Rouge depuis I943. vice-président (195I)

MARCEL JUNOD, docteur en médecine: délégué du Comité international de la Croix-Rouge de I935 à 1946 (1952)

ERNEST NOBS, ancien conseiller fédéral (1952)

\section{Direction :}

MM. ROGER GALLOPIN, directeur exécutif

JEAN S. PICTET, directeur des Affaires générales

DAVID DE TRAZ, directeur exécutif-ađjoint

ÉDOUARD DE BONDELI, sous-directeur

CLAUDE PILLOUD, sous-directeur des Affaires générales

\section{Secrétariat général:}

M. JEAN DUCHOSAL, secrétaire général

1 Les années indiquées dans les parenthèses désignent les dates de nomination des membres du Comité international. 


\title{
REVUE INTERNATIONALE DE LA CROIX-ROUGE
}

\author{
TRENTE-SIXIEME ANNEE - $N^{\circ} 427$ \\ JUILLET 1954 \\ *
}

SOMMAIRE

COMITE INTERNATIONAL DE LA CROIX-ROUGE

Activités récentes ................ ${ }_{529}$

Extraits du Rapport annuel (1er janvier au 31 décembre 1953) . . 532

90 ANNIVERSAIRE DE LA CONVENTION DE GENÈVE

En marge du « Congrès de Genève » : Conférence internationale

des Sociétés de secours (10 et 11 août 1864) . . . . . 543

Sur l'esprit de service, par Jean-G. Lossier . . . . . . . . . 557

\section{NOTES ET DOCUMENTS}

Conférence internationale pour la neutralisation du Service de santé militaire en campagne : Procès-verbaux des séances (8-22 août 1864) (suite et fin) (hors-texte) . . . . . . . . 573

Avant le "Congrès de Genève " : Lettres de Henry Dunant et de Gustave Moynier . . . . . . . . . . .

\section{A TRAVERS LES REVUES \\ * \\ BULLETIN INTERNATIONAL DES SOCIÉTÉS DE LA CROIX-ROUGE}

(Voir sommaire, page 605)

Paratt à la fin de chaque mois

Edrtrur : Comité international de la Croix-Rouge

RÉDACTEUR RESPONSABLE : Louis Demolis

PrIx, franco, un an : Fr. 20.- ; le numéro Fr. 2,-. Administration et publicité : CICR, 7, av. de la Paix, Genève. Téléphone 3330 60. Compte de chèques I. 1767 


\title{
S UP PLÉME N TS DE LA REV UE
}

SOMMAIRE

\section{EN LANGUE ANGLAISE}

July 1954, No. 7, Vol. VII :

International Committee of the Red Cross : The Geneva Convention in International Law, by Henri Coursier, Legal Department of the ICRC.

Balance Sheet as on December 31, 1953. - General Income and Expenditure Account as on December 31, 1953.

*

\section{EN LANGUE ESPAGNOLE}

Julio 1954, No 7, Vol. VI:

Comité Internacional de la Cruz Roja : El Convenio de Ginebra en el Derecho Internacional, por Henri Coursier, Miembro del Servicio Juridico del CICR.

Balance del CICR al 31 de Diciembre de 1953. - Cuenta general de Gastos e Ingresos del CICR al 31 de Diciembre de 1953.

Comunicado.

\author{
* \\ EN LANGUE ALLEMANDE \\ Juli 1954, Nr. 7, Band V:
}

Internationales Komitee vom Roten Kreuz: Die Genfer Konvention im Internationalen Recht, von Henri Coursier, Mitglied der Rechtsabteilung des IKRK.

Bilanz per 31. Dezember 1953. - Allgemeine Rechnung der Ausgaben und Einnahmen per 31. Dezember 1953. 


\section{Votre Banque...}

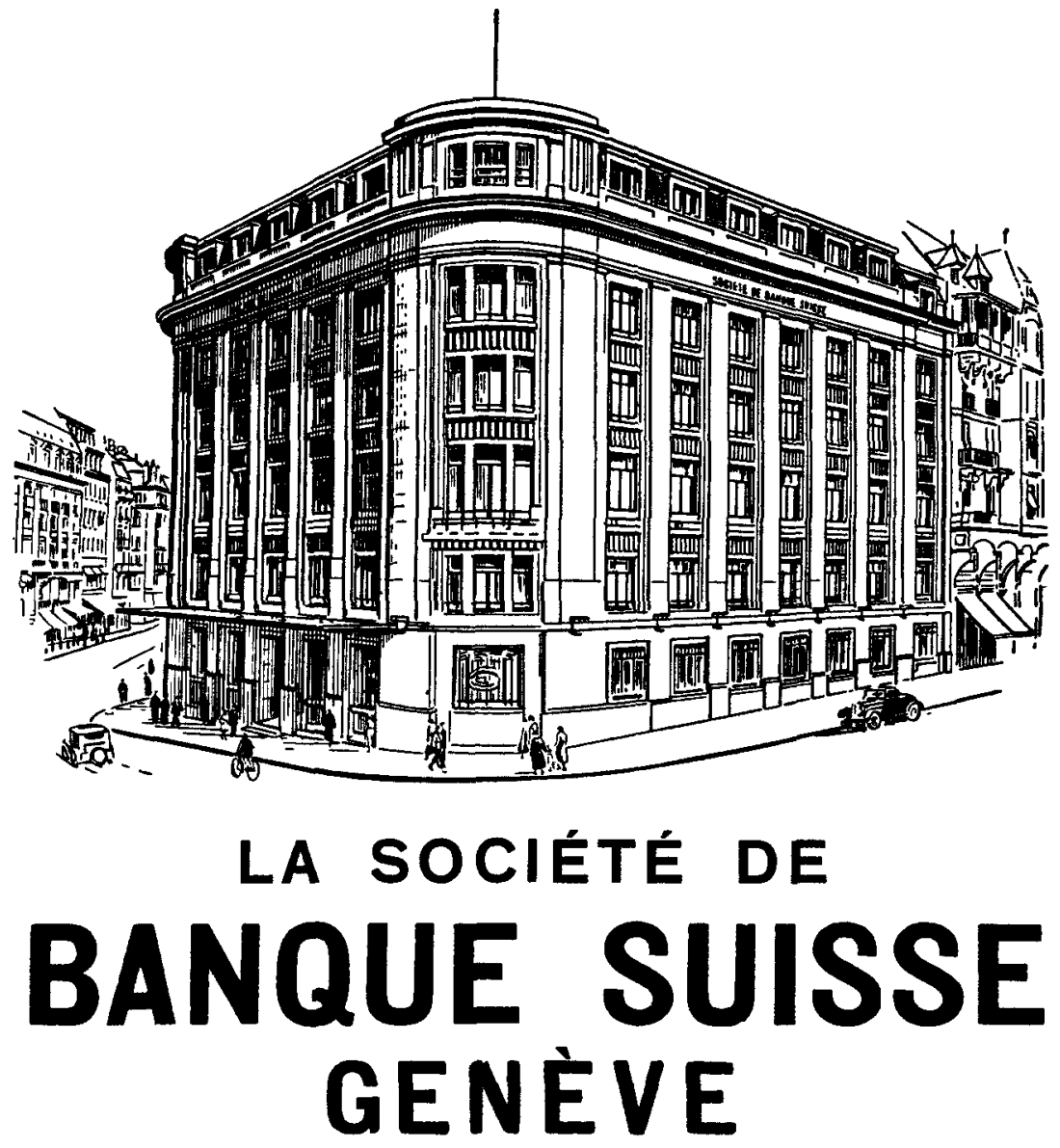

2, rue de la Confederation

BALE ZURIOH ST-GALL LAUSANNE NEUCHATEL OHAUX-DE-FONDS SCHAFFHOUSE BIENNE LONDRES NEW-YORK

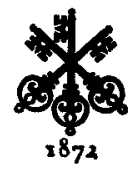

CAPITAL ET RÉSERVES FRS 226 MILLIONS 


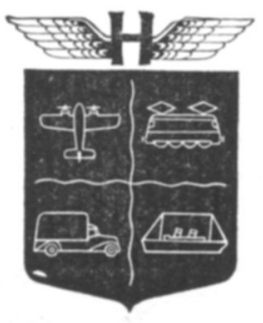

Compagnie d'Assurances Générales

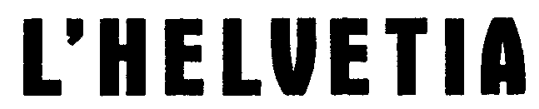

ST-GALL (Suisse)

Fondée en 1858

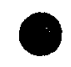

\section{ASSURAICES TRAISPORTS}

et

REASSURAhCES dans toutes les Branches

Capital social ( $1 / 2$ versé)

Total des moyens de garantie

Primes brutes 1952
Francs sulsses 10.000.000 58.000.000 45.000.000 
Nous élaborons nos spécialités pharmaceutiques avec le concours des instituts et cliniques en vue d'après les méthodes de recherches scientifiques les plus modernes

Les préparations portant la marque

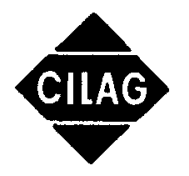

sont partout appréciées des médecins pour leur qualité éprouvée, constante et sûre 


\section{SOCIÉTÉ FIDUCIAIRE ROMANDE OFOR S.A.}

- Revisions et organisations comptables

- Administration et liquidation de sociétés

- IMPOTS : Conseils par spécialiste

9, RUe D'italie - Genteve TELEPHONE 24 32 91/3

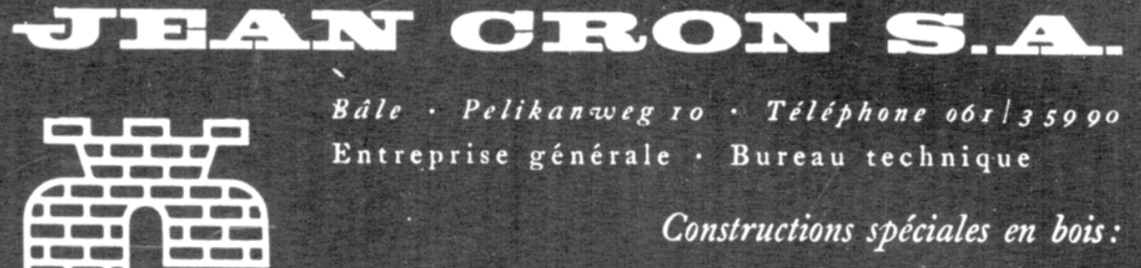

강

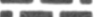

Baraquements, maisons d'habitation, maisons provisoires, hangars agricoles, dépôts pour denrées et marchandises, bâtiments de bureau, hôpitaux, garages, entrepôts, hangars pour avions ect.

Laboratoires

SAPOS S.A.

\section{Spécialités}

pharmaceutiques

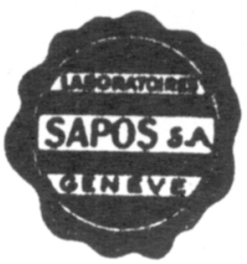

11, RUE DE LA NAVIGATION

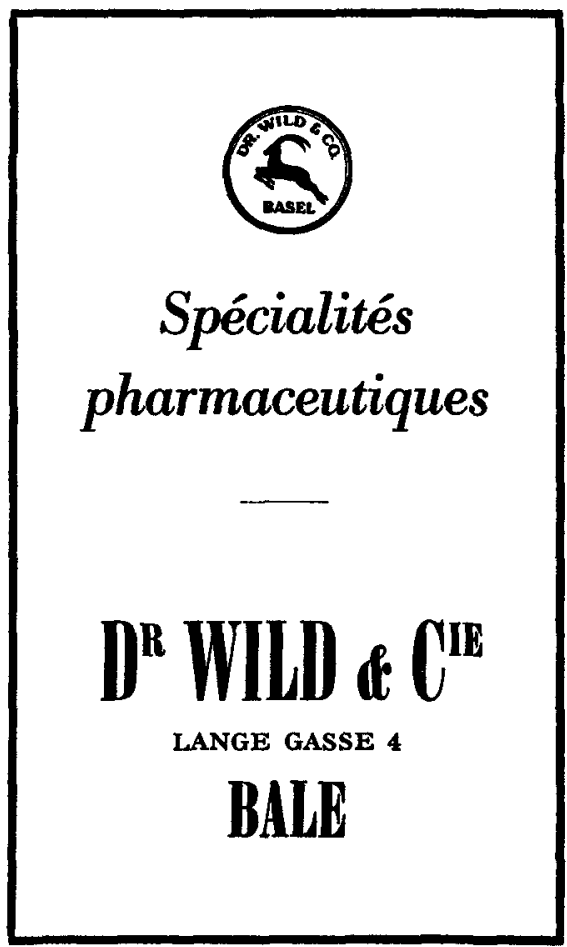




\section{Ruderset \& Dubois}

AGENCE DE VOYAGES

PLACE CORNAVIN 16 - GRNEVE - TÉLEPHONE 326000

Grand Tourisme - Excursions SERVICE REGULIRR GENEVE-COTE D'AZUR par autocars de luxe - Hotesse - Bar - Toilottos TRANSPORTS ROUTIERS INTERNATIONAUX

\section{ENTREPRISE GÉNÉRALE \\ ÉLECTRICITÉ ET TÉLÉPHONE}

\section{FORNASARI}

5, avenue Henri-Dunant

GENEVE

Tól. 244034

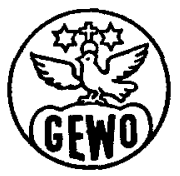

«GEWO ”

LA MARQUE DE CONFIANCE POUR SPECIALITES PHARMACEUTIQUES PREPAREES SUR LA BASE DE NOS PROPRES RECHERCHES

LES FILS D'ED. GEISTLICH S. A. POUR L'INDUSTRIE CHIMIQUE SECT. PHARMACEUTIQUE

WOLHUSEN / SUISSE 

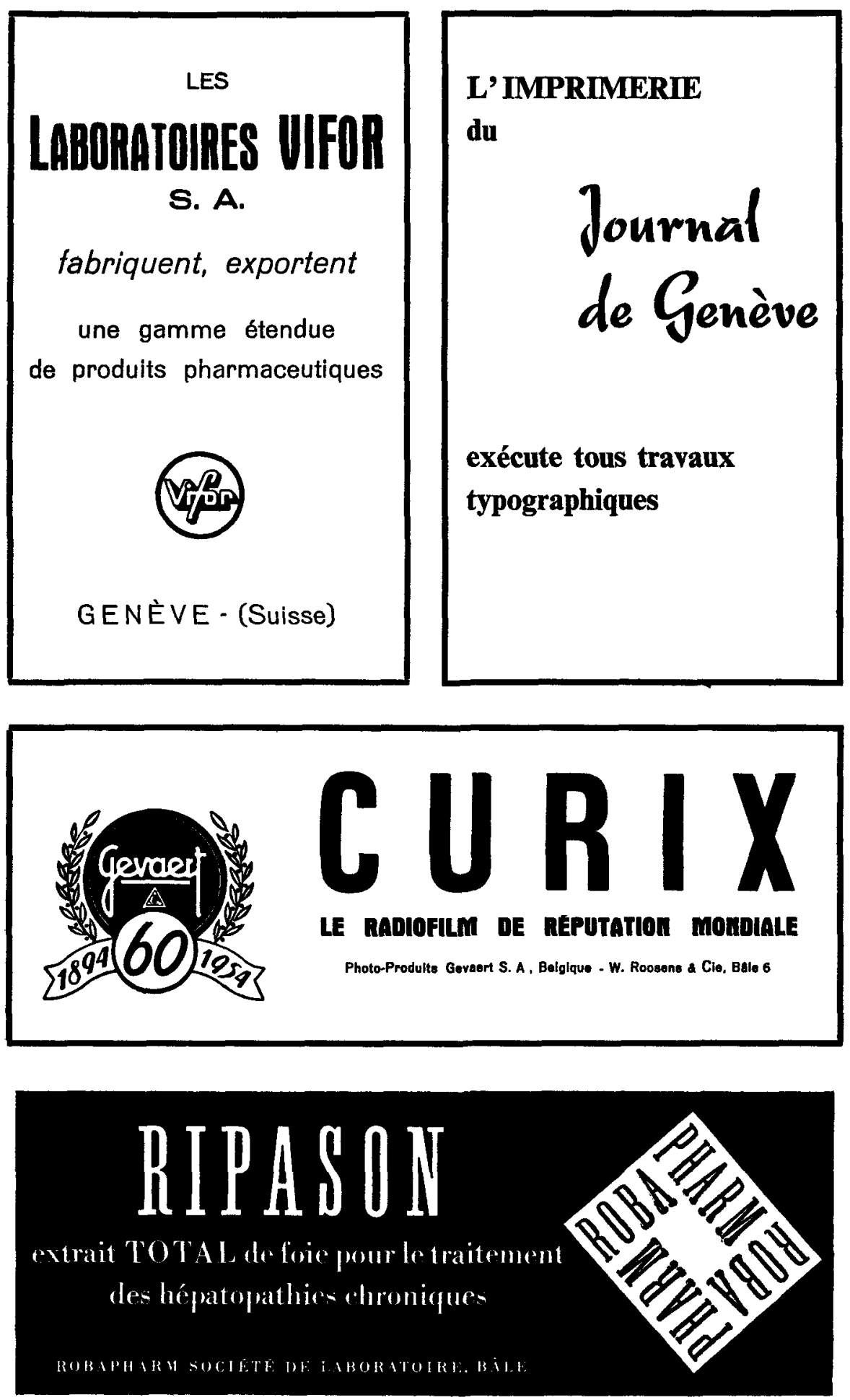


\section{S.A. anct B. SIEGFRIED ZOFINGUE (SUISSE) • FONDÉE EN 1873}

Fabrication, depuis quatre-vingts ans, de produits chimiques et pharmaceutiques d'une qualité irréprochable à l'intention des médecins et des pharmaciens

Exportation dans tous les pays des médicaments synthétiques préparés conformément aux prescriptions des diverses pharmacopées

Dérivés chimiques purs à disposition de l'industrie et pour les recherches scientifiques

Spécialités pharmaceutiques éprouvées, préparées selon les données les plus récentes de la médecine 


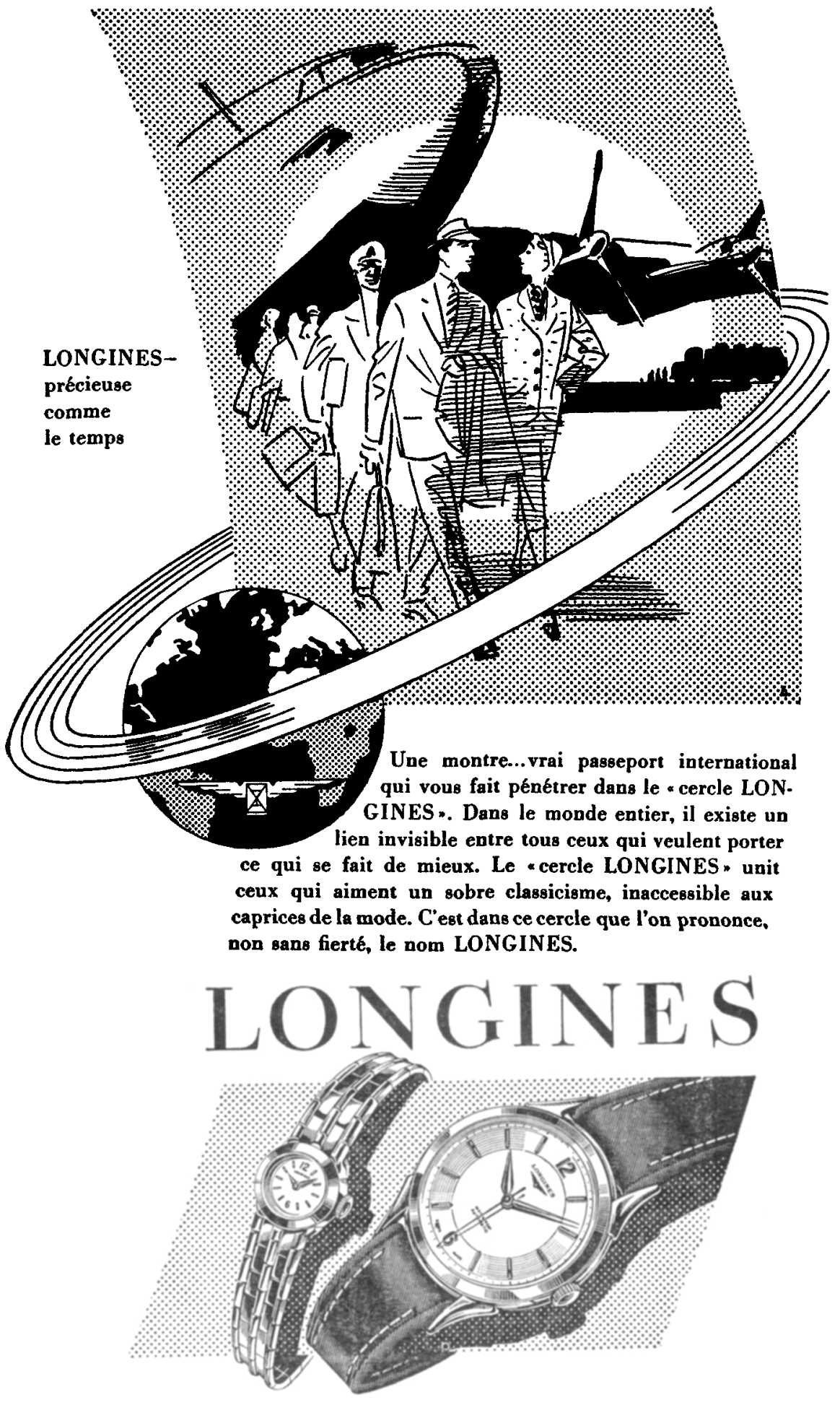




\section{IAIT SUISSg}

Marque

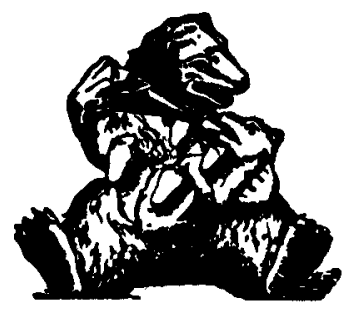

"A L'OURS"

PREPARE PAR

SOCIÉTÉ LAITIĖRE DES ALPES BERNOISES

STALDEN, Emmenthal, SUISSE

\section{SOCIÉTÉ ANONYME \\ FIDUGIAIRE SUISSE}

$\begin{array}{ll}\text { BALE } & \text { St. Albananlage 1 } \\ \text { Zurich } & \text { Talstrasse } 80 \\ \text { Genève } & \text { Rue du Mont-Blanc 3 } \\ \text { Lausanne } & \text { Place St-François 14 B }\end{array}$

REVISIONS • EXPERTISES • ORGANISATIONS QUESTIONS FISCALES 


\section{BONNES ADRESSES EN SUISSE}

\section{LA RÉSIDENCE}

Florissant 11

GENEVE

Hôtel - Restaurant - Bar

Granda of petits salons pour réceptions 200 lits 60 salles de bains

TEléphone dans toutes les chambres

Deux tennis - Parc pour autos Arrangements pour familles Tel. 241380 (8 lignes)

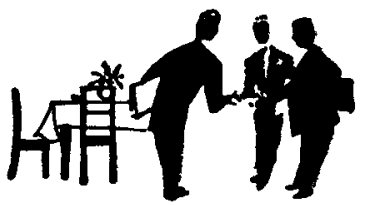

Pour leurs conférences, ils se réunissent toujours au

\section{Buffet de la tare à Perne}

\section{F. E. KRÄHENBUHL}

Téléphone 23421
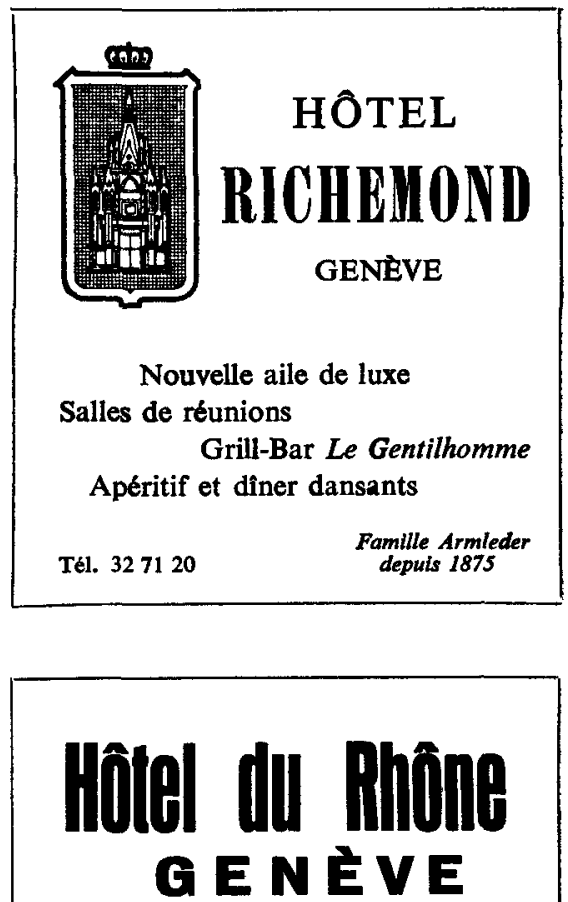

Le plus récent, construit en 1950 , en plein centre. Situation tranquille au bord du Rhône, quai Turrettini. 200 chambres, toutes avec salle de bain, douches ou cabinet de toilette, radio et safe. Restaurant, café-glacier, bar, nombreuses salles de conférences bureaux à la journée avec dactylo à disposition.

Garage dans la maison Agence de voyages Coiffeur, fleuriste, chemisier - maroquinier.

Prix à partir de fr. 16,-, chambre, petit-déjeuner et service compris.

Tol. 327040 Tólogr.: RHohotel

\section{Institut Mingerfa Zurich Próparation a Soctlon commerclale l'Universite of Cours pour l'Ecole Palytechnique aldes-médecins}

